\title{
Follow-up Observation of Aortitis Syndrome
}

\author{
Shigenori Morooka, M.D., Iwao Ito, M.D., Hisomu Yamaguchi, M.D., \\ Tadanao TAKeda, M.D., and Yoshimi SAIto, M.D.
}

\begin{abstract}
SUMMARY
Follow-up observations have been made on 64 cases of aortitis syndrome for an average period of 6.6 years ( 3 months to 15 years). Thirteen cases died during the observation period. The survival rate at the end of the 10th observation year was estimated to be $62 \%$. Main causes of death were congestive heart failure and cerebrovascular accident. The prognosis depended mainly upon the grade of hypertension. The daily life of patients was more or less limited in $49 \%$ but improved slightly in the course. Symptoms and signs of active inflammatory process have been improved by steroid hormones. In some cases, long-term treatment with a small dose of steroid hormone was required for several years.
\end{abstract}

\section{Additional Indexing Words:}

Aortitis syndrome Takayasu's arteritis Prognosis Steroid hormone Survival rate Secondary hypertension

\begin{abstract}
ORTITIS syndrome ${ }^{4)}$ is a clinical entity caused by non-specific arteritis A (Takayasu's arteritis) of the aorta, its major branches and the pulmonary arteries. It involves mostly young woman in the Orient.

The clinical pictures, ${ }^{2)-7)}$ pathological findings ${ }^{81-11)}$ and epidemiology of the disease have been described by several authors and etiological investigations ${ }^{12-17)}$ have also been reported. However, little has been known about the natural history ${ }^{18)-20}$ of the disease or the result of long-term steroid therapy. The purpose of this paper is to present these informations obtained from 64 cases of aortitis syndrome.
\end{abstract}

\section{Materials And Methods}

Follow-up observation was made on 64 of 72 patients with aortitis syndrome who were admitted to the Second Department of Internal Medicine, University of Tokyo from 1954 to 1971 . They consisted of 53 females and 11 males, and the period of observation ranged from 3 months to 15 years, the average being 6.6 years. The average age at the time of admission was 31.2-year-old. Forty-three cases visited the outpatient clinic for reevaluation. In the remaining 21 cases, the informations were obtained through questionaire.

From the Second Department of Internal Medicine, Faculty of Medicine, University of Tokyo, Japan.

Received for publication March 4, 1972. 
Eighteen women experienced pregnancy after the onset of this disease and obtained 31 babies. Surgical treatment was performed in 10 cases; Bypass teflon graft from the aorta to the renal artery was placed in 6 cases, and 5 of them were improved (blood pressure became normal in 3 cases), though another case was unchanged. Of the remaining 4 cases, one received an operation of bypass from the aorta to the left common carotid artery by which her symptoms were improved. One case received thromboendarterectomy for bilateral carotid arteries followed by improvement of her symptoms but she died of cerebral softening due to carotid artery occlusion after 3 years. A case with an aneurysm of the common carotid artery and a case with aneurysms of the abdominal aorta died during operation.

\section{RESUlts}

1. Survival rate curve

During the observation period, 13 of 64 cases died. This means that the mortality in an average of 6.6 years was $20 \%$. The mortality was $63.5 \%$ ( 7 of 11 cases) in the males and $11.3 \%$ (6 of 53 cases) in the females. Death in the lst year of observation occurred in 7 of 64 cases, indicating that the survival rate at the end of the lst year was $89 \%$. In the 2nd year, death occurred in 1 of the surviving 57 cases, indicating that the survival rate for the 2nd year was $98 \%$. This means that the survival rate at the end of the 2 nd observation year was $87 \%$ (89\% multiplied by $98 \%$ ) against the original total cases. The survival rate at the end of each observation year calculated by such a way is plotted in Fig. 1, illustrating that the survival rate at the end of the

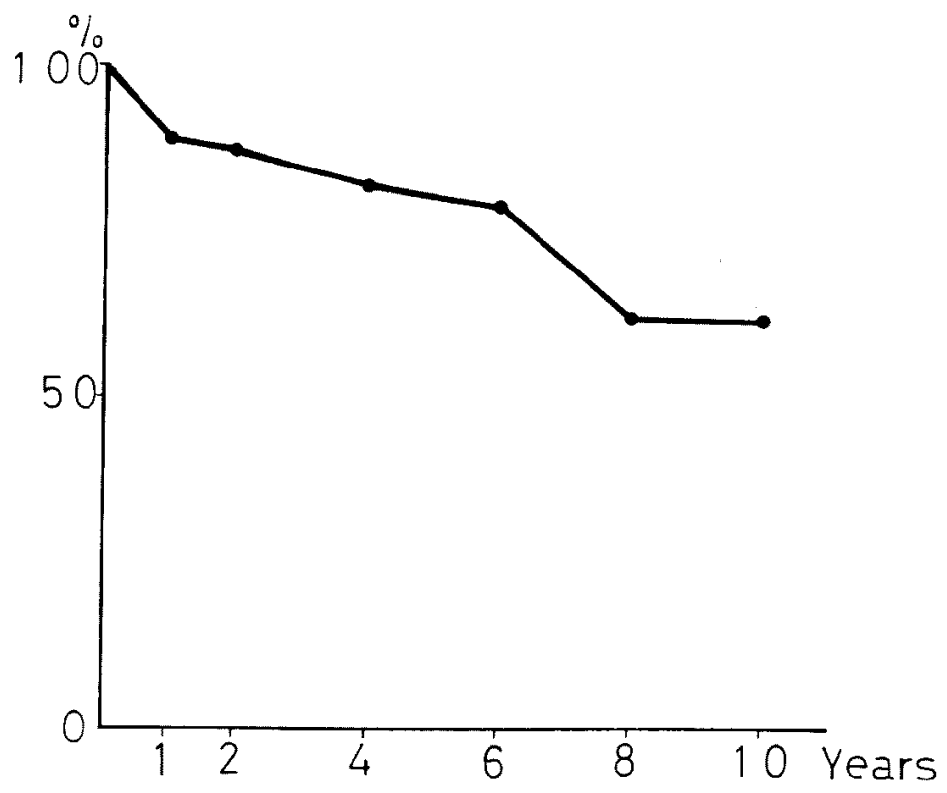

Fig. 1. Gurve of survival rate. 
Table I. Causes of Death

\begin{tabular}{l|c}
\multicolumn{1}{c}{ Cause of Death } & Number of Cases \\
\hline Congestive heart failure & 4 \\
Cerebral hemorrhage & 3 \\
Cerebral softening & 2 \\
Operation & 2 \\
Accident (with congestive heart failure) & 1 \\
Respiratory muscular paralysis & 1
\end{tabular}

10th year is estimated to be $62 \%$.

2. The causes of death

Table I shows the causes of death in the 13 fatal cases. Four cases died of congestive heart failure, 3 of cerebral hemorrhage, 2 of cerebral softening, 2 of operation, 1 of suicide related to intractable heart failure and 1 of respiratory muscular paralysis. The most frequent causes of death were congestive heart failure and cerebrovascular accidents. The age of the death was 21 to 30 -year-old in 5 cases and 31 to 40 -year-old in 6 cases (Table II).

Table II. Ages at the Time of Death

\begin{tabular}{c|c}
\hline Age & Number of Cases \\
\hline $21 \sim 30$ & 5 \\
$31 \sim 40$ & 6 \\
$41 \sim 50$ & 1 \\
$51 \sim$ & 1
\end{tabular}

Of 36 patients with hypertension, in whom systolic pressure of $150 \mathrm{~mm} . \mathrm{Hg}$ or higher, or diastolic pressure of $90 \mathrm{~mm} . \mathrm{Hg}$ or higher was noted in any extremity, 8 patients $(22 \%)$ developed cerebral hemorrhage and $5(14 \%)$ developed heart failure, while only $2(7 \%)$ of 28 patients without hypertension developed cerebral softening, suggesting that the prognosis of the patients is greatly influenced by coexisting hypertension.

3. Morbidity in daily life

Among the causes which more or less restricted the activity in daily life of the patients were hypertension (36 cases), aortic insufficiency (13), cerebral hemorrhage (8), heart failure (5), cerebral softening (2), visual disturbance (8), and perforation of nasal septum (2). As mentioned above, heart failure and cerebrovascular accidents constituted the main causes of death.

In 6 of 8 cases with visual disturbance, blood pressure in ocular fundus was abnormally lowered. One of 2 cases with Takayasu's fundus (peripapillary 
arterio-venous anastomoses) developed blindness of the both eyes and another developed blindness of the right eye in the course. The remaining 4 cases revealed microaneurysms and venous dilatation in ocular fundus, and 2 of them suffered from narrowing of visual field.

In 2 of 8 cases, visual disturbance was associated with hypertensive retinal changes (Keith-Wagener IV). One of them developed blindness of the both eyes due to retinal detachment, and another had narrowing of visual field. The perforation of nasal septum occurred in 2 cases and seemed to be due to ischemia resulting from bilateral common carotid artery lesions.

The daily activity of the patients was classified into 4 groups according to the grade of its restriction and the severity of complaints. Patients of group 1 had no complaint and no limitation in their daily life, those of group 2 had minor complaints such as dizziness, headache and numbness of the extremities but these symptoms did not interfere with their normal daily activity, those of group 3 had more marked symptoms such as pain, exertional palpitation, dyspnea and fatigability which more or less disabled them, and those of group 4 were seriously ill because of heart failure, blindness or hemiplegia. Fig. 2

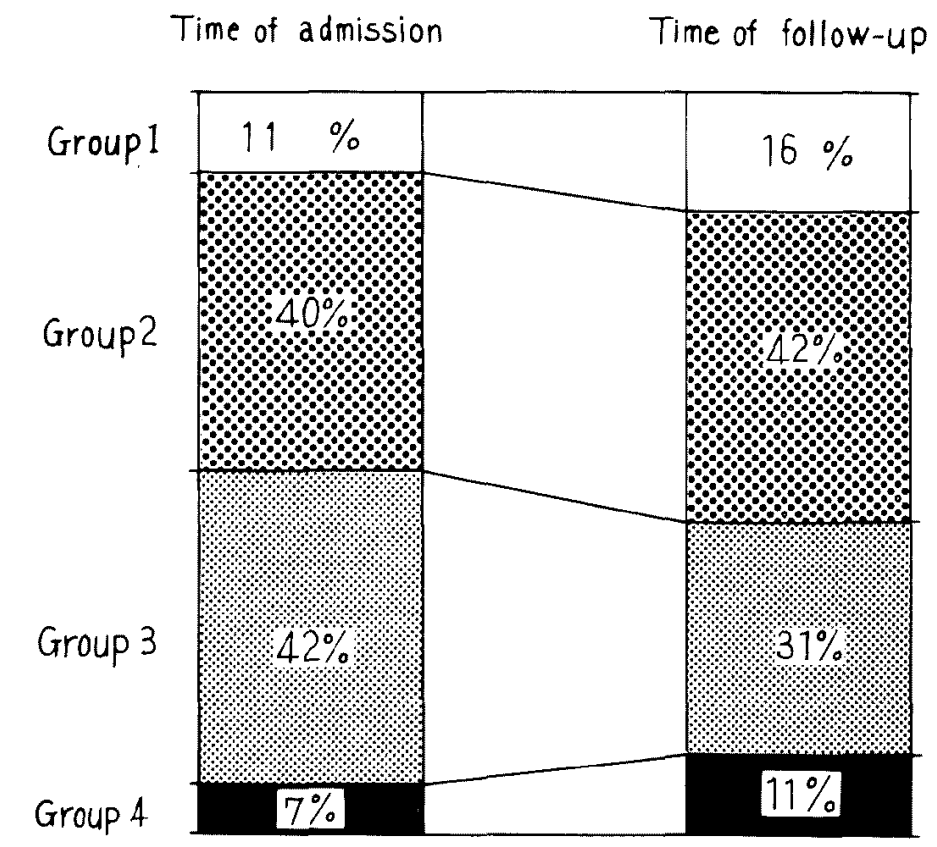

Fig. 2. Activity of daily life in 45 cases at the time of admission and the time of follow-up study.

Group 1: no complaint and no limitation of activity

Group 2: minor complaints but no limitation of activity

Group 3: some degree of limitation of activity

Group 4: seriously disabled 


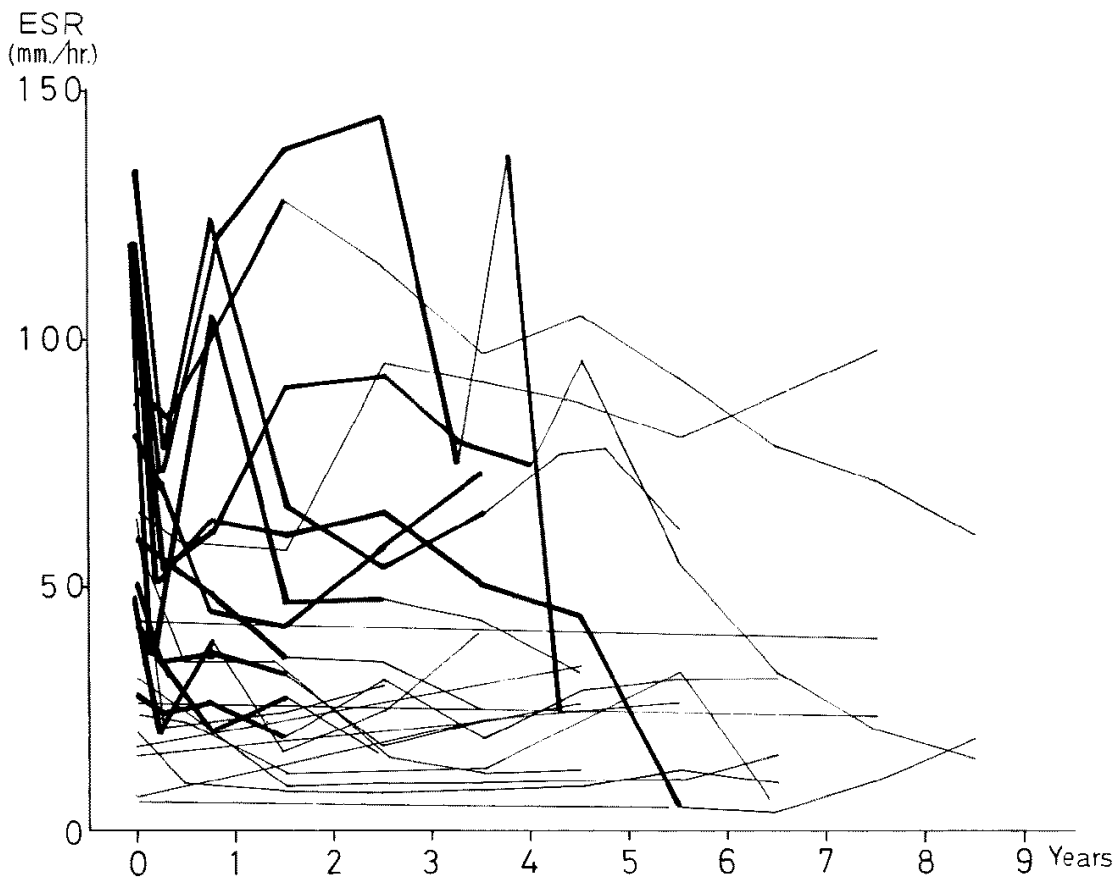

Fig. 3. The changes of ESR in the course. The period of steroid therapy is expressed by thick lines.

demonstrates the comparison of the activity at the time of admission and that at the time of follow-up study in 45 surviving patients who were observed for more than 1 year. At the time of admission, 5 cases $(11 \%)$ were classified in group $1,18(40 \%)$ in group $2,19(40 \%)$ in group 3 , and $3(7 \%)$ in group 4. As a whole, tendency to improved activity rather than deterioration predominated at the time of follow-up study, although the ratio of group 4 increased from $7 \%$ to $11 \%$.

4. Effects of steroid hormones and follow-up of several clinical findings

Treatment with steroid hormones was made in 32 cases with subjective symptoms and/or laboratory findings due to active arteritis. This resulted in a subjective improvement in 23 cases $(72 \%)$. The treatment was particularly effective to pain in the neck, chest or back ( 7 cases), headache ( 7 cases), dizziness ( 7 cases), fever ( 5 cases), and fatigability (4 cases). Improvement of visual acuity was obtained in 3 cases, and lowering of blood pressure was noted in a case with renovascular hypertension. In association with these effects, laboratory evidences of active inflammation such as accelerated erythrocyte sedimentation rate (ESR), positive G-reactive protein (CRP), hypergammaglobulinemia and elevated anti-aorta antibody titer were also improved. Fig. 3 illustrates the time course of ESR in 23 cases, of which 12 


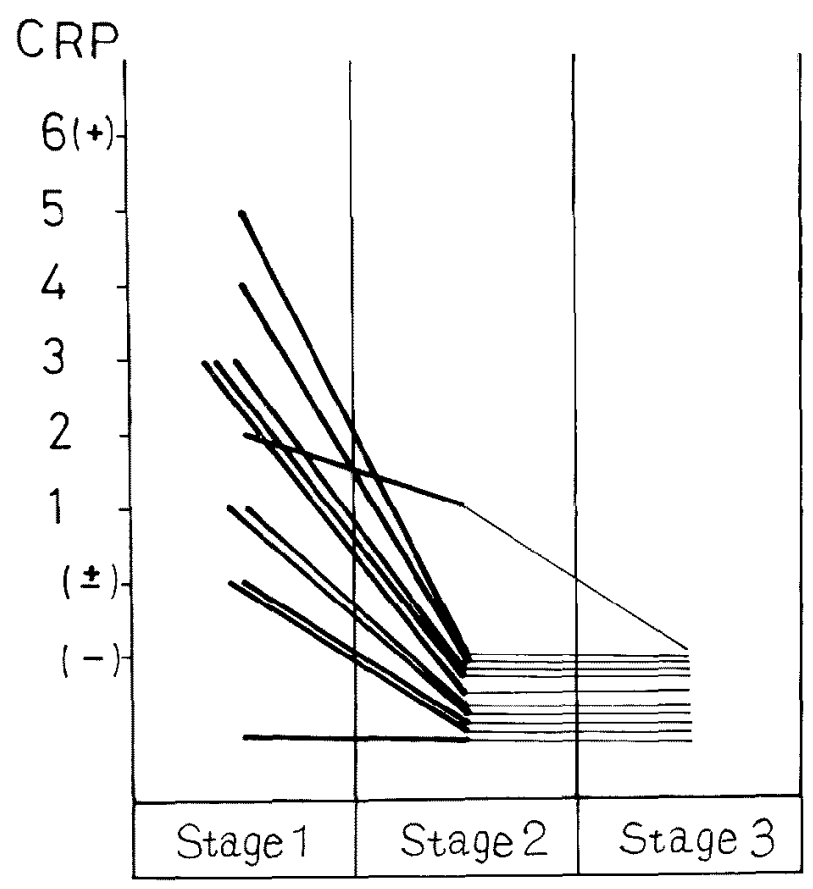

Fig. 4. The changes of CRP in the course.

Stage 1: On admission; Stage 2: During steroid therapy; Stage 3: After steroid therapy.

The average interval between stages 1 and 2 was 2.5 years, and that between stages 2 and 3 was 2.6 years.

received steroid therapy. In cases with a markedly accelerated ESR over $60 \mathrm{~mm}$. per hour, administration of prednisolone was started with a daily dose of $20 \mathrm{mg}$. on an average, reducing the dosage to 5 to $10 \mathrm{mg}$. daily within 6 months. They often required a long-term administration of a small dose of steroid in order to maintain the therapeutic effect, and in some of them it could be withdrawn after 4 to 5 years. In cases with less marked acceleration of ESR below $60 \mathrm{~mm}$. per hour, steroid therapy could be discontinued usually within 1 to 3 years. In cases with no or only slight acceleration of ESR, in which steroid was not used, it remained unchanged throughout the course of 3 to 9 years. No serious side effect of steroid therapy was recognized, although moon face was noted in 11 cases, acne in 1 and transient glycosuria in 1.

Fig. 4 demonstrates the time course of GRP in 11 cases treated with steroid hormones. In 10 of them, it became negative following a steroid therapy of 2.5 years on an average, and remained negative for an average period of 2.6 years after discontinuance of steroid. CRP was negative in 8 of 11 cases in which steroid was not used, and it remained negative after an average period of 6.0 years. 


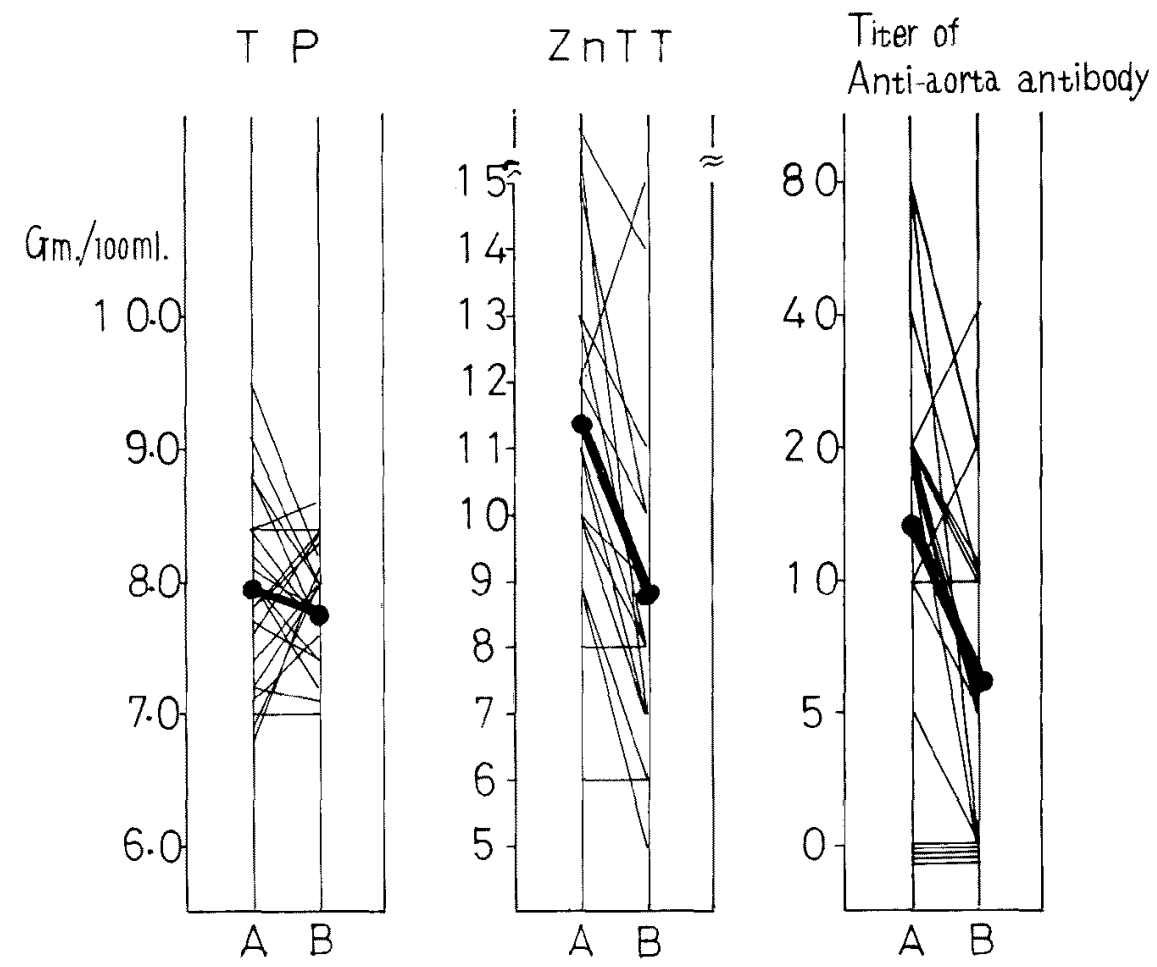

Fig. 5. The changes of serum total protcin (TP), zinc sulfate turbidity test $(\mathrm{ZnTT})$ and titer of anti-aorta antibody.

A: values at the admission, B: values at the follow-up study. Thick lines indicate average values.

In Fig. 5 are included the cases treated and those not treated with steroid. The average value of zinc sulfate turbidity test in 16 cases was 11.4 units on admission and decreased to 8.8 units at the time of follow-up study. The difference was significant $(\mathbf{P}<0.005)$. The fall in the titer of circulating antiaorta antibody determined by complement fixation test was also significant $(\mathrm{P}<0.001)$. The average serum total protein level decreased from 7.9 to $7.5 \mathrm{Gm} . / 100 \mathrm{ml}$., but the difference was not significant $(\mathrm{P}>0.1)$. Blood urea nitrogen exceeding $30 \mathrm{mg} . / 100 \mathrm{ml}$, was found in no case on admission and in none of 18 cases in which it was reexamined at follow-up study. No particular changes in the peripheral blood picture and the serum total cholesterol level were noted during the course.

Follow-up observation of blood pressure in 39 surviving cases revealed that hypertension persisted in all cases even after suppression of active arteritis, except for 3 cases in which normal blood pressure was restored by an operation. Among the cases, which were normotensive on admission, 2 cases developed hypertension during the follow-up period. 
Number

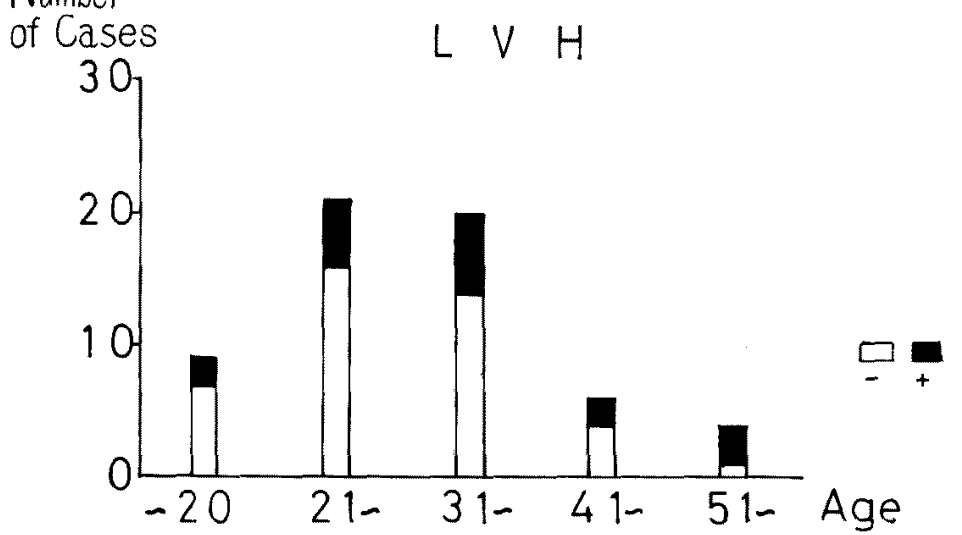

Number $\quad C T R$

of Cases

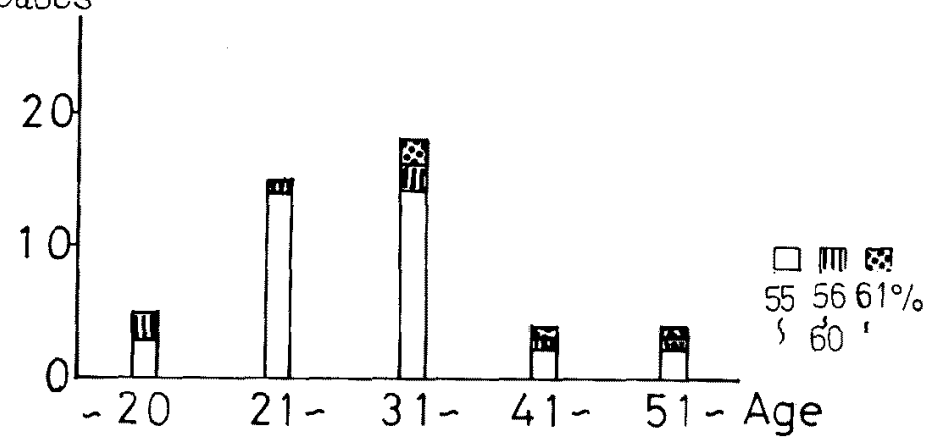

Fig. 6. Frequency of evidences of left ventricular hypertrophy in EGG (upper) and an increased cardiothoracic ratio in chest $\mathrm{X}$-ray (lower) in patients of each decade of age.

The findings of Fig. 6 that evidences of left ventricular hypertrophy in EGG and an increased cardiothoracic ratio in chest X-ray were noted with a higher frequency in older patients than in younger patients may be related, at least partly, to the persisting hypertension. Fig. 7 shows the results of followup analyses of ECG findings in 21 cases. In 10 cases with hypertension, the occurrence of left ventricular hypertrophy pattern increased progressively with time, reaching $60 \%$ at the 10 th year, while it remained low $(27 \%)$ in 11 cases without hypertension.

Two examples of long-term steroid therapy are presented in the following.

Case 1. M. M., a 20-year-old female. The patient was admitted complaining of severe headache. She had acute nephritis at 5-year-old and an operation of sinuitis at 15-year-old. She suffered from vertigo and noted the absence of pulsation of the left radial artery by herself at 18-year-old. Her blood pressure on admission 
Vol. 13
No. 3

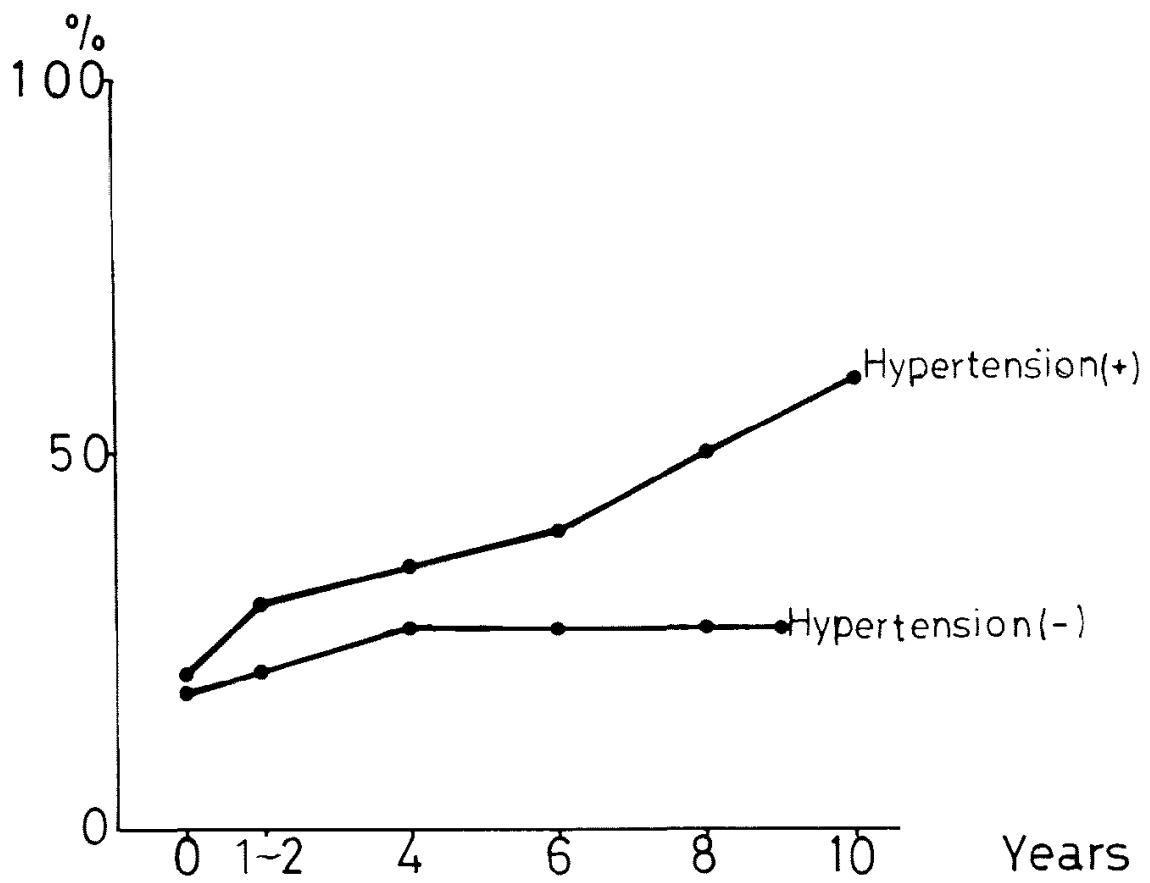

Fig. 7. Relationship between the frequency of ECG pattern of left ventricular hypertrophy and the duration of the disease.

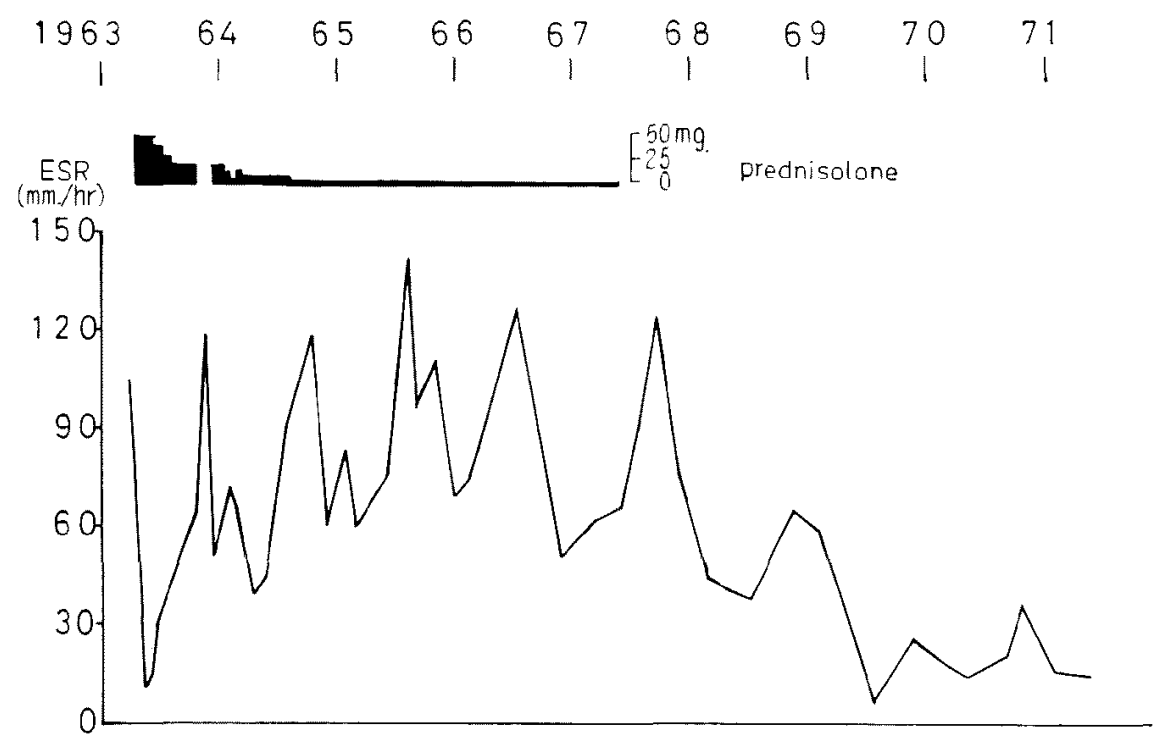

Fig. 8. The course of case 1 . 
was $130 / 60 \mathrm{~mm} . \mathrm{Hg}$ in the right arm, 94/68 in the left arm, 178/72 in the right leg and not measurable in the left leg. Laboratory data were as follows: ESR $119 \mathrm{~mm}$./hour, CRP $4(+)$, STS negative, hemoglobin $74 \%$ (Sahli), white blood cell count 4,000, ZnTT 13.2, serum total protein $8.8 \mathrm{Gm} . / 100 \mathrm{ml}$., A/G ratio 0.8 , $\alpha_{2}$-globulin $6.4 \%, \gamma$-globulin $25.2 \%$, and anti-aorta antibody titer $20 \times$. In aortogram, stenoses were found in the superior mesenteric artery, right carotid artery, left subclavian artery and left vertebral artery. The patient was diagnosed to have aortitis syndrome of active stage, and was treated with dexamethasone, the daily dosage of which was $6.75 \mathrm{mg}$. initially and was decreased gradually (Fig. 8). Her headache disappeared and weak pulse of the left radial artery became palpable. As ESR decreased to $10 \mathrm{~mm}$./hour and CRP became negative, she was discharged after 2 and a half months. Moon face and acne were noted at the time of discharge. During the subsequent 7 months, she was given $20 \mathrm{mg}$./day of prednisolone and the dosage was gradually decreased to discontinue it. However, soon after the withdrawal she complained of vertigo and headache again, ESR was accelerated to $113 \mathrm{~mm}$./hour, and CRP became $2(+)$. Therefore, dexamethasone was resumed with a daily dose of $2.0 \mathrm{mg}$. and symptoms disappeared shortly. The dosage was decreased to $0.5 \mathrm{mg}$. daily in 8 months and the same dosage was continued for 3.5 years. In this period she was able to work as a housewife and obtained a normal baby. The treatment was discontinued at age of 24 years when ESR decreased to $53 \mathrm{~mm}$./hour and CRP became negative. Since then, she has been living for 3 years without receiving steroid and her laboratory data have been maintained within normal limits.

Case 2. S.S., a 27-year-old female. Ten months prior to admission, the patient suffered from high fever of $40^{\circ} \mathrm{C}$, fatigue and nausea, accompanied by an accelerated ESR of more than $100 \mathrm{~mm}$./hour and CRP of $3(+)$. In spite of subjective improvement she was admitted for detailed examinations. At the time of admission, her body temperature was $37.5^{\circ} \mathrm{C}$, blood pressure was $100 / 60 \mathrm{~mm} . \mathrm{Hg}$ in the right arm, $120 / 65$ in the left arm, 120/50 in the left leg and 125/60 in the right leg. Laboratory data were as follows; Hemoglobin 74\% (Sahli), white blood cell count 5,300,

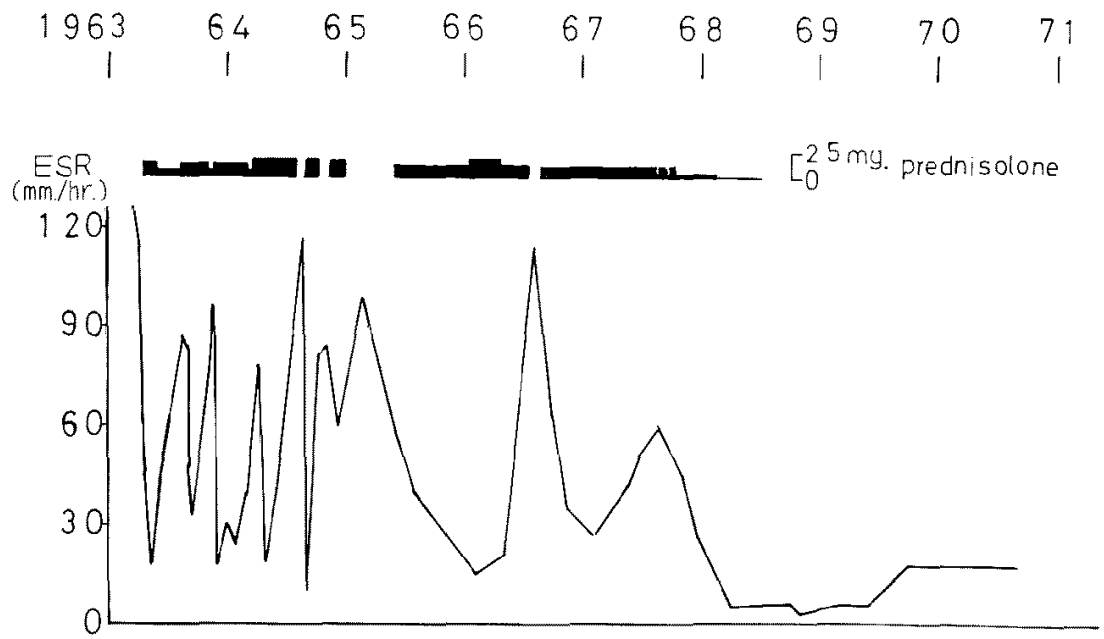

Fig. 9. The course of case 2 . 
ESR $122 \mathrm{~mm}$./hour, CRP 3(+), STS negative, ASLO 250, serum total protein $8.0 \mathrm{Gm} . / 100 \mathrm{ml}$, $\mathrm{A} / \mathrm{G}$ ratio $1.2, \alpha_{2}$-globulin $12 \%, \gamma$-globulin $34.3 \%$, and anti-aorta antibody titer $80 \times$. A small old tuberculous focus was found in the chest X-ray film. In aortogram, localized stenoses were found in the right carotid artery, right subclavian artery and left subclavian artery. The patient was diagnosed to have aortitis syndrome of active stage, and prednisolone was used with a daily dose of $20 \mathrm{mg}$. for 2 months. The dosage was then decreased gradually to $5 \mathrm{mg}$./day. As ESR decreased to $18 \mathrm{~mm}$./hour and CRP became negative, she was discharged with no complaints and no side effect of steroid. During the subsequent course, she occasionally complained of palpitation, abdominal pain and pain of the upper extremities and the daily dosage of prednisolone was changed from 5 to $15 \mathrm{mg}$. The steriod therapy had to be continued for 5 years, because its withdrawal resulted in aggravation of her symptoms and laboratory data. Thereafter, however, she has been living well for 4 years without use of steroid hormone, and laboratory data have remained normal.

\section{Discussion}

The reports of aortitis syndrome have been made mainly from the oriental countries such as Japan, ${ }^{2)-41,7)}$ India, ${ }^{15)}$ Ceylon, ${ }^{21)}$ Malaysia, ${ }^{22)}$ Thailand, ${ }^{23)}$ Korea $^{24)}$ and China ${ }^{25)}$ and occasionally from other countries. However, there have been only a few reports ${ }^{18)-20)}$ which described the natural history of this disease.

Inada et al. ${ }^{19)}$ followed the course of 71 cases for 4 years and a month and found that 12 cases died. The present study disclosed that there was a mortality rate of $20 \%$ during an average observation period of 6.6 years and that the survival rate at the 10 th year after the admission was estimated to be $62 \%$.

The causes of death consisted mainly of heart failure $(5$ cases, one of which had an accident) and cerebrovascular accidents ( 5 cases). It was demonstrated that the severity of secondary hypertension was of particular importance for the prognosis of the disease. Among 36 patients with hypertension, congestive heart failure occurred in $14 \%$ and cerebral hemorrhage in $28 \%$, while among 28 patients without hypertension cerebral softening occurred in only $7 \%$. After 10 years duration of the disease, ECG evidences of left ventricular hypertrophy were noted in $60 \%$ of the cases with hypertension but in $27 \%$ in those without hypertension. In addition, the mortality rate in the observation period was $25 \%$ in the cases with hypertension, while it was $14 \%$ in those without hypertension.

The hypertension associated with aortitis syndrome may be attributed to one or more of the following factors: it is frequently due to renal artery stenosis; stenotic changes in the descending aorta may cause an elevated blood pressure of the upper half of the body; systolic hypertension may occur as the result of aortic insufficiency or widespread sclerosing lesions of the aorta; steno- 
sis or occlusion of main branches of the aortic arch may result in compensatory hypertension in the other artery arising from the aorta. In the present series, recurrence of active arteritis was scarcely experienced during the chronic inactive stage. Therefore, the distribution of the arterial lesions contributing to hypertension seems to be fixed in the early active stage of the disease. In other words, the prognosis of the patients may become decisive within the initial period following the onset of the disease. This is supported by the fact that death occurred more frequently in younger patients than in older patients. Consequently, the importance of early suppressive treatment against the active inflammatory process should be emphasized. Surgical operation ${ }^{26)}$ should be considered for the treatment of fixed hypertension.

Takayasu et al. ${ }^{20)}$ observed the daily life of 24 patients with this disease and found that 17 cases were restrained of their work at the time of admission but that 13 cases were improved after the discharge. The present study also demonstrated a tendency, though not significant, to improved activity of the surviving patients. This may be explained by disappearance of the symptoms due to active arteritis such as fever, pain and fatigue, or by adaptation phenomenon or development of collateral circulation.

Steroid therapy has been proved to be most effective for suppression of active arteritis. In general, the period needed for withdrawal of steroid hormones was proportional to the severity of the evidences of active inflammation. Long-term administration of a small dose of steroid hormones for several years was required in some cases in order to maintain the therapeutic effects.

\section{REFERENCES}

1. Ueda, H., Ito, I., and Saito, Y.: Jap. J. Int. Med. 15: 239, 1965.

2. Committee Report: Jap. Heart J. 9: 76, 1968.

3. Ueda, H., Morooka, S., Ito, I., Yamaguchi, H., Takeda, T., and Saito, Y.: Jap. Heart J. 10: $277,1969$.

4. Shimizu, K. and Sano, K.: J. Neuropath. Clin. Neurol. 1: 37, 1951.

5. Ask-Upmark, E.: Acta Med. Scandinav. 149: 161, 1954.

6. Sen, P. K., Kinare, S. G., Engineer, S. D., and Parulkar, G. B.: Brit. Heart J. 25: 610, 1963.

7. Nakao, K., Ikeda, M., Kimata, S., Nitani, H., Miyahara, M., Ishimi, Z., Hashiba, K., Takeda, Y., Ozawa, T., Matsushita, S., and Kuramochi, M.: Circulation 35: 1141, 1967.

8. Oota, K.: Trans. Soc. Path. Jap. 30:680, 1940.

9. Danaraj, T. J. and Ong, W. H.: Circulation 20: 856, 1959.

10. Nasu, T.: Angiology 14: 225, 1964.

11. Saito, Y., Ito, I., Yamaguchi, H., Takeda, T., Morooka, S., Hirota, K., and Ueda, H.: J. Jap. College Angiology 2: 77, 1971.

12. Ito, I.: Jap. Circulat. J. 30: 75, 1966.

13. Ueda, H., Saito, Y., Ito, I., Yamaguchi, H., Sugiura, M., and Morooka, S.: Jap. Heart J. 8: 4,1967 .

14. Ueda, H., Saito, Y., Morooka, S., Ito, I., Yamaguchi, H., and Sugiura, M.: Jap. Heart J. 9: $573,1968$. 
15. Kinare, S. G,: J. Path. 100:69, 1970.

16. Muñoz, N. and Correa, P.: Am. Heart J. 80: 319, 1971.

17. Numano, F. and Shimamoto, T.: Am. Heart J. 81 : 591, 1971.

18. Strachan, R. W.: Quart. J. Med. 33: 57, 1964.

19. Inada, K., Yoshida, K., Katsumura, T., Shiga, S., and Shirahige, T.; Blood and Vessel 2: 581,1971 .

20. Takayasu, M. and Ishikawa, K.: Jap. J. Clin. Med. 26: 63, 1968.

21. Thenabadu, P. N., Rajasuiya, K., and Wickremasenghe, H. R.: Brit. Heart J. 32: 181, 1970.

22. Ooi, B. S., Chen, B. T. M., Yu, S. F., Toh, C. C. S., and Khoo, O. T.: Austral. New Zealand J. Med. 1: 32, 1971 .

23. Vinijchaikul, K.: Am. J. Med. 43: 15, 1967.

24. Lee, K. S., Sohn, K. Y., Hong, C. Y., Kong, S. R., and Gerg, K.: Acta Paediat. Scandinav. 56: $526,1967$.

25. Wan, H. and L. Sheng, L.: Chinese Med. J. 81: 526, 1962.

26. Inada, K., Katsumura, T., Hirai, J., and Sunada, T.: Arch. Surg. 100: 220, 1970. 